\title{
Circulating Progenitor Stem Cells Are Important Biomarkers of Chondrogenesis and Osteogenesis: Employment in Diagnosis and Treatment Follow Up
}

\author{
Maria Teresa Valenti ${ }^{1}$, Monica Mottes ${ }^{2 *}$ and Luca Dalle Carbonare ${ }^{1}$ \\ ${ }^{1}$ Department of Medicine, Internal Medicine Section, Verona, Italy \\ ${ }^{2}$ Department of Neurosciences, Biomedicine and Movement Sciences; Biology \& Genetics Section, Verona, Italy
}

\begin{abstract}
Adult multipotent mesenchymal stem cells (MSCs) arouse great scientific interest because of their many possible clinical applications. Besides the therapeutic potential for tissue regeneration and repair, they also offer new opportunities as diagnostic tools. For diagnostic purposes peripheral blood represents an easily accessible source of circulating MSCs which show similar characteristics compared to bone marrow MSCs. Dysfunctions in chondrogenic and osteogenic differentiation of MSCs are involved in the genesis of age-related degenerative disorders as osteoarthritis and osteoporosis. Altered expression profiles of specific transcription factors may be monitored in the ex vivo analysis of patients' peripheral blood MSCs; they represent important biomarkers for diagnosis. Furthermore, changes in MSCs expression profiles induced by pharmacological treatments are useful biomarkers for therapy followup. MicroRNAs also play a crucial role in chondrogenic and osteogenic differentiation of progenitor cells; differentially expressed microRNAs have been associated with osteoarthritis and osteoporosis, respectively. MicroRNAs can be recovered from blood, urine, and synovial fluid and represent useful supplemental tools for the diagnosis of these degenerative disorders.
\end{abstract}

In conclusion, circulating MSCs can be obtained by a non-invasive approach and provide an "ex vivo" source for monitoring various differentiation pathways in normal and pathological conditions; thus they represent a remarkable resource for various clinical applications.

Keywords: Circulating MSCs; Osteogenesis; Chondrogenesis; microRNAs; Biomarkers; Gene expression analysis

\section{Abbreviations}

bALP: Bone Alkaline Phosphatase; BM-MSC: Bone Marrow Mesenchymal Stem Cell; BMP: Bone Morphogenetic Protein; CMP: Cartilage Matrix Protein; COMP: Cartilage Oligomeric Protein; ECM: Extra Cellular Matrix; ESC: Embryonic Stem Cells; FGF: Fibroblast Growth Factor, GH: Growth Hormone; GR: Glucocorticoid Receptor; HSC: Hematopietic Stem Cell; IGF1: Insuline-Like Growth Factor 1; miRNA: microRNA, MNC: Mononuclear Cell; OA: Osteoarthritis; OC: Osteocalcin; OP: Osteoporosis; OPN: Osteopontin; OSX: Osterix (SP7 transcription factor); PB-MSC: Peripheral Blood Mesenchymal Stem Cell; PTH: Parathormone; RUNX2: Runt-Related Transcription Factor 2; SOX\#: SRY-box\#; SPARC: Osteonectin; TGF- $\beta$ : Transforming Growth Factor- $\beta$; TNF- $\alpha$ : Tumor Necrosis Factor-a; WNT\#: WinglessType MMTV Integration Site Family Member\# 1; ZA: Zoledronic Acid

\section{Background}

\section{Stem cells biology, circulating stem cells}

The great interest surrounding stem cells biology is prompted by their remarkable capacities of multipotency and self-renewal. Embryonic Stem Cells (ESCs) are pluripotent, since they can differentiate into the three germ layers and are capable of unlimited undifferentiated proliferation in vitro. Adult multipotent stem cells, primarily isolated from bone marrow, can be distinguished in two different types: Hematopoietic Stem Cells (HSCs) committed to differentiate into blood cells, and stromal stem cells which are named Bone Marrow Mesenchymal Stem Cells (BM-MSCs), capable of differentiating into several lineages of mesodermal and non-mesodermal origin. Other sources of MSCs have been identified through the years, including placenta, adipose tissue, dental pulp, synovium, endometrium, breast milk [1]. Circulating MSCs can be recovered from umbilical cord blood as well as from adult peripheral blood [2]. Peripheral blood MSCs (PBMCSs) have shown similar characteristics in terms of multipotency and proliferation, compared to BM-MSCs. Unfortunately their number is very low in physiological conditions: approximately $1: 10^{8}$ mononuclear cells (MNCs). Appropriate enrichment methods must be devised for their selection and utilization. Potential clinical applications of such cells, obtainable by a non-invasive approach, are very interesting; the possibility to make them proliferate and differentiate in vitro discloses also therapeutic applications for tissue regeneration.

\section{Mesenchymal stem cells in cartilage and bone}

BM-MSCs are the precursors of cartilage cells (chondrocytes), bone cells (osteoblasts), as well as fat cells (adipocytes). Thus chondrogenesis and osteogenesis arise from the same progenitor cells and are subject to complex regulation by interplaying agents such as systemic hormones, e.g. growth hormone (GH), insulin-like growth factor 1 (IGF-1), parathormone $(\mathrm{PTH})$, estrogens et al., and local factors such as fibroblast growth factors (FGFs), transforming growth factor beta (TGF-beta),

*Corresponding author: Monica Mottes, Department of Neurosciences, Biomedicine and Movement Sciences; Biology \& Genetics Section, Strada Le Grazie, 837134 Verona, Italy, Tel: +390458027184; E-mail: monica.mottes@univr. it; luca.dallecarbonare@univr.it; mariateresa.valenti@univr.it

Received April 05, 2018; Accepted April 20, 2018; Published April 30, 2018

Citation: Valenti MT, Mottes M, Carbonare LD (2018) Circulating Progenitor Stem Cells Are Important Biomarkers of Chondrogenesis and Osteogenesis: Employment in Diagnosis and Treatment Follow Up. J Stem Cell Res Ther 8: 425 doi: 10.4172/2157-7633.1000425

Copyright: (c) 2018 Valenti MT, et al. This is an open-access article distributed under the terms of the Creative Commons Attribution License, which permits unrestricted use, distribution, and reproduction in any medium, provided the original author and source are credited. 
bone morphogenetic proteins (BMPs), WNT signaling pathways. Common chondrogenic/osteogenic precursors express the master transcription regulator Runt-related transcription factor-2 (RUNX2). SRY-box9 (SOX9), the chondrogenic differentiation marker, warrants chondrogenesis versus osteogenesis by negatively regulating RUNX2 [3]. Two other SOX family members (SOX5 and SOX6) are regulated by and co-expressed with SOX9; their combined activity is required for transformation of progenitor cells into early chondroblasts. In the endochondral ossification process, which characterizes long bones development, chondrocytes provide a template through proliferation, terminal differentiation to the hypertrophic state and finally apoptosis, whereby calcified cartilage is replaced by osteoblast-produced osteoid. In the articular cartilage, a resilient tissue which covers the ends of long bones at the joints, chondrocytes remain quiescent; they are responsible for the production and homeostatic maintenance of extracellular matrix (ECM) components. Structural ECM components, which determine articular cartilage mechanical and frictional properties, are: collagens (mainly type II collagen), proteoglycans (including aggrecan, decorin, biglycan, and fibromodulin), non-collagenous proteins, such as cartilage oligomeric protein (COMP), cartilage matrix protein (CMP), fibronectin. The primary matrix degrading enzymes involved in cartilage turnover are metalloproteinases and cathepsins. In healthy articular cartilage, balanced degradation and synthesis by chondrocytes ensure tissue homeostasis. The osteogenic commitment of pluripotent BM-MSCs is controlled by RUNX2, which in turn activates the osteogenic master regulator OSTERIX (OSX) [4]. RUNX2 expression, however, must be suppressed for the accomplishment of osteoblastic maturation, as demonstrated in animal models and in human pathological conditions [5,6]. The differentiation process implies the expression of downstream genes coding for specific osteoblastic proteins such as collagen type I, osteocalcin (OC), osteonectin (SPARC), osteopontin (OPN), bone sialoprotein (BSP), which are the organic constituents of bone ECM. Bone alkaline phosphatase (bALP) is also produced by osteoblasts and is involved in the mineralization of bone matrix (Figure 1) [7].

Epigenetic factors, such as microRNAs (miRNAs) are also involved in BM-MSCs commitment, differentiation and survival. These small noncoding RNAs act as negative modulators of gene expression. They interfere with translation by binding to partially complementary sequences in the 3'UTR sites of target mRNAs. Circulating miRNAs can be found in different biological fluids such as serum/plasma, urine, and synovial fluid. MiRNAs can be secreted by MSCs and transported by extracellular vesicles, e.g. exosomes, which in turn will undergo endocytotic internalization to recipient cells. Thus exosomes play important roles in cellular communications which occur in bone modeling and remodeling [8]. MiRNA profiling in exosomes isolated from BM-MSCs at various stages of osteogenic differentiation allowed the identification of differentially expressed miRNAs at each stage [9]. When administered to uncommitted BM-MSCs, in the absence of any other osteogenic supplement, exosomes deriving from each stage were able to induce osteogenic differentiation. However, only exosomes released by cells at the late stages of differentiation could induce the mineralization process. The regulatory effects on osteogenesis exerted by miRNAs are certainly entitled to further investigation.

\section{Dysfunctions of MSCs in osteoarthritis and osteoporosis}

Osteoarthritis (OA), a degenerative musculoskeletal disease of the joints, is characterized by cartilage alteration, bone remodeling at subchondral level, osteophytosis and pain. Chondrocytes, which are surrounded by the cartilage ECM, have a limited ability in intrinsic regeneration [10]. In joints, subchondral bone supports the articular cartilage and, when microfractures occur, BM-MSCs are released in order to repair damaged cartilage. In addition to subchondral bone, also synovium contains MSCs. However, MSCs harvested from OA joints are not able to proliferate and differentiate properly [11] probably because of the altered inflammatory environment [12,13]. It has been reported that a subpopulation of progenitor cells, found in OA patients' cartilage, exhibits an early senescent phenotype [14]. This subpopulation, expressing CD271 antigen, also lacks proliferative capacity; senescence of progenitors and loss of proliferation potential may contribute to OA pathophysiology [15]. Importantly, macrophages residing in the synovia, which produce pro-inflammatory cytokines, act as negative regulators of chondrogenesis [16,17]. Decreased expression levels of SOX9, the master gene of chondrogenesis, have been observed in MSCs of OA patients. In addition, dysfunctions in proteasome and autophagic machinery have been reported in chondrocytes of $\mathrm{OA}$ patients [18]. Epigenetic factors may as well influence chondrogenesis. Altered expression patterns of specific miRNAs [19], as well as altered chromatin remodeling [20] can impair the commitment and differentiation of MSCs in OA patients' joints.

Osteoporosis (OP) is a common degenerative skeletal disease affecting most postmenopausal women and aged people in general. Alterations in BM-MSCs differentiation are involved in its genesis. OP is the consequence of an imbalance between bone formation and bone resorption. It has been reported that alterations in osteogenic differentiation of progenitor cells prevent bone formation and impair the physiological process of bone remodeling. MSCs' commitment is regulated by a network involving various transcription factors and signaling molecules [21]. Among them, several WNT family members (e.g. WNT1, WNT6, WNT10A, and WNT10B) play an important role for the osteogenic commitment of MSCs. Epigenetic inhibition of WNT pathway in OP animal models suppresses osteogenic differentiation [22]. Tumor Necrosis Factor-alpha (TNF- $\alpha$ ) associated with estrogen deficiency (which is considered the most important cause of OP), impairs osteogenic differentiation by reducing antioxidant defenses [23]. The pro-osteogenic action of transcription factor RUNX2 is often affected by factors inducing osteopenia conditions. An elevated production of glucocorticoids impairs bone microarchitecture causing bone loss and skeletal deterioration. It has been reported that glucocorticoids antagonize RUNX2 during osteogenic differentiation. This effect is actually due to a physical interaction between RUNX2 and activated glucocorticoid receptor (GR) [24]. As for epigenetic factors, miRNA 204 and miRNA 211 are endogenous repressors of RUNX2 [19]; MiRNA-29a, on the contrary, can reduce glucorticoid-induced bone loss by rescuing RUNX2 activity [24]

\section{Gene expression profiles of circulating MSCs as markers of bone /cartilage homeostasis in health and disease}

Several years ago, we developed a method for isolating MSCs from peripheral blood (Figure 2) [25]. In brief, cMSCs are obtained from $50 \mathrm{ml}$ of peripheral blood using an antibodies cocktail to deplete other blood cells. The protocol entails two subsequent density gradient centrifugations. In the first, mononuclear cells from heparinized peripheral blood are obtained. Then, mononuclear cells are mixed with $200 \mu \mathrm{l}$ of an antibodies cocktail containing bi-specific antibody complexes against red blood cells (glycophorin A) and CD3, CD14, CD19, CD38, CD66b positive cells (hematopoietic cells). Hematopoietic cells crosslinked with red blood cells are then discarded by an additional gradient centrifugation; the phenotype of isolated cells is thereafter determined. Evaluation of surface markers (cluster 


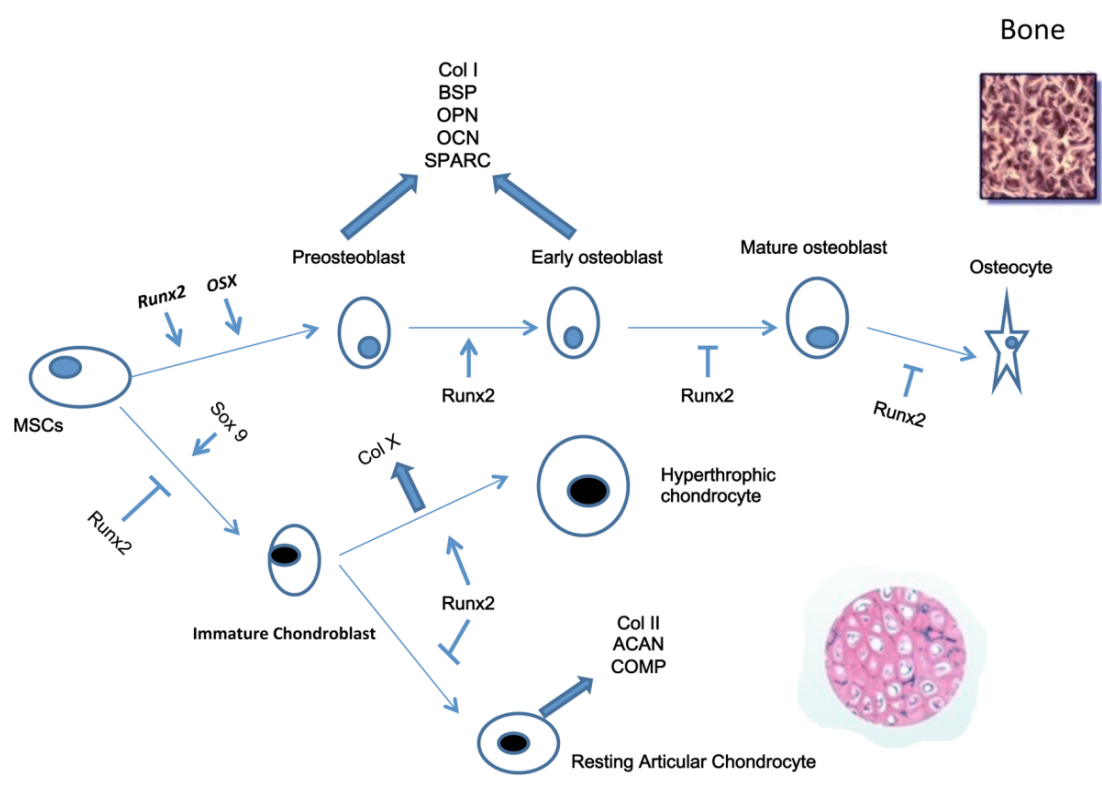

Cartilage

Figure 1: Regulators of osteoblast and chondrocyte differentiation steps. In the osteogenic differentiation of bipotent BM-MSC, RUNX2 and, sequentially OSX, play a crucial role, promoting the expression of bone ECM proteins. For further osteoblast maturation, RUNX2 must be downregulated. SOX9 expression drives the chondrogenic differentiation pathway; in the endochondral ossification process RUNX2 is necessary for chondrocyte maturation to hypertrophic chondrocyte, inducing $\mathrm{COLX}$ and other ECM components production.

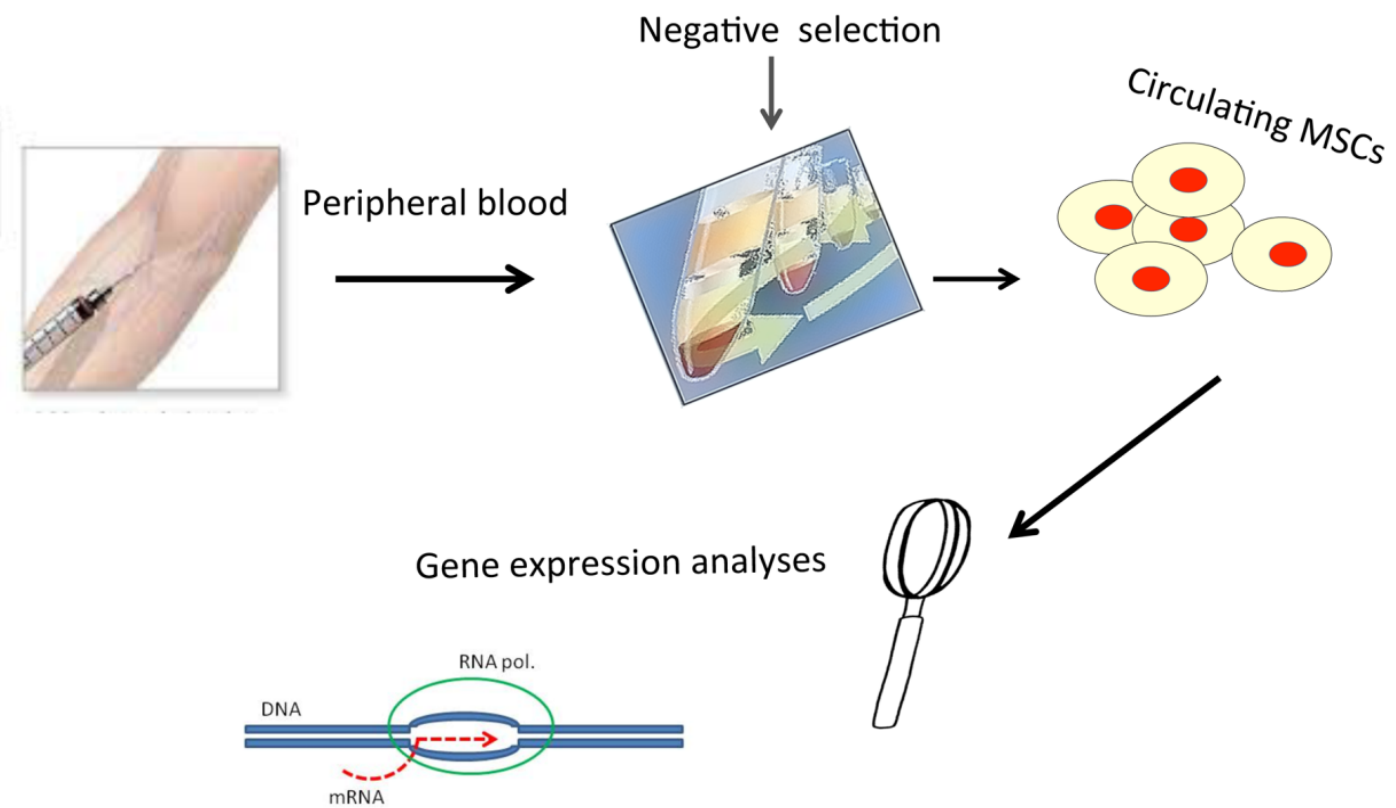

Figure 2: Recovery of circulating MSCs. Circulating MSCs can be obtained from peripheral blood by a two-step method including an enrichment of mononuclear cells followed by depletion of unwanted cells (Ref.\#25). Thereafter cMSCs can be used to perform gene expression analyses.

differentiation) of PB-MSCs is important for diagnostic purpose. Usually, isolated PB-MSCs with a percentage of positive markers (such as $\mathrm{CD} 105$ and $\mathrm{CD} 73)>60 \%$ and with a low $\%$ of hematopoietic negative markers (such as CD3, CD19, CD14, CD45) are considered suitable for diagnostic analyses [6]. By this method we were able to define the
PB-MSCs profiles during commitment and differentiation in normal subjects. Thereafter we have identified up- or down-regulated genes in disorders affecting bone [6,26-28], as well as cartilage [29]. In PBMSCs from OP patients, we observed a reduced expression of RUNX2 as well as an enhanced expression of the adipogenic transcription factor 
PPARG2 [26]. Interestingly, data obtained by analyzing PB-MSCs confirmed the positive correlation between the atherogenic lipid profile and osteopenia features in OP post-menopausal patients [30] and suggested PB-MSC as important biomarkers for early diagnosis. An ex vivo analysis of zoledronic acid (ZA) therapy in OP patients was made by evaluating gene expression profiles in PB-MSCs [31]. The analysis highlighted the anabolic activity of ZA and also suggested the use of these biomarkers for therapy follow-up. Recently, we found a reduced expression of SOX9, the chondrogenesis master gene, in PB-MSCs obtained from patients affected by OA [29]. This observation suggests SOX9 expression as a useful PB-MSC biomarker for the identification of cartilage dysfunction. In addition, we showed an enhanced expression of SOX9 in patients PB-MSCs, upon treatment with clodronate correlating with the reduction of clinical symptoms. Gene expression profiling is a useful tool also for OA monitoring.

\section{Expression profiles of circulating miRNAs as disease biomarkers}

The expression profiles of specific miRNAs can also be associated with $\mathrm{OA}$ and $\mathrm{OP}$ features, respectively. They may represent useful supplemental tools for the diagnosis of these degenerative disorders. MiR-181-5p, which targets COL2A1 in chondrocytes and is associated with cartilage degeneration, can be recovered from peripheral blood [32]; it may therefore represent a marker for screening OA patients [33]. Members of the miR-183 cluster (miR-183/-182/-96), that can inhibit the osteogenic differentiation of BM-MSCs, may as well be used as diagnostic biomarkers for OP. The presence of these miRNAs actually increases in exosomes released by aged bone [34]. Similarly, miR-31$5 p$, involved in different steps of bone differentiation, can be found in exosomes; it has been suggested as a biomarker for osteoporosis [35]. Interestingly, a study describing the human miRNome in OP and OA patients highlighted different circulating miRNAs as biomarkers for the screening of these complex diseases [36].

\section{Are PB-MSCs useful diagnostic tools?}

Circulating blood cells may somehow be regarded as signallers of alterations occurring in the macro- or micro-environment of organs and tissues. This observation suggests the use of circulating cells as "surrogate tissue" for diagnostic analyses [37]. In particular, PBMSCs may be useful tools directing towards the identification of early pathological conditions related to skeletal diseases as well as towards a correct diagnosis. Besides the traditional cytofluorimetric analyses of PB-MSCs, new technologies are requested to investigate these cells at different molecular levels (i.e. epigenome, transcriptome, methylome). We may overcome their paucity, a factual drawback, thanks to technological advancements. Gene expression analyses performed by array approaches or by Next Generation Sequencing (NGS) are effective, despite some limitations due to the background noise [38]. Certainly, data obtained by PB-MSCs analyses have to be confirmed and supported by additional clinical and biochemical observations.

\section{Potential therapeutic applications of PB-MSCs}

Besides the diagnostic applications discussed above, therapeutic applications for PB-MSCs (e.g. autologous/heterologous transplantation) appear very promising, provided that efficient protocols for isolation and in vitro culture expansion are devised. There are several examples of MSC-based therapy attempts for bone and cartilage defects, both in animal models $[39,40]$ and in humans $[41,42]$. Most studies have employed BM-MSCs. Rare reports of studies in animal models employing PB-MSCs have been published to our knowledge [43]. The laborious steps necessary for their isolation and self-renewal in vitro may have discouraged their employment so far. However, there is an increasing expectation on cell-based therapies employing MSCs for the treatment of degenerative and inflammatory diseases. Exploring all accessible sources of such precious cells is of paramount importance.

\section{Conclusion}

Circulating mesenchimal stem cells show characteristics of proliferation and multipotency similar to those of BM-MSCs. They are normally present in minimal concentrations; it is known that their number increases after mobilization from $\mathrm{BM}$ in response to injury $[44,45]$.

PB-MSCs can be obtained by a non-invasive approach and provide an ex vivo source for monitoring various differentiation pathways in normal and pathological conditions. They therefore represent a remarkable resource for various clinical applications as well as for preclinical research in order to indicate how and where molecular pathways get disrupted in degenerative diseases. Altered expression profiles of commitment-specific mRNAs and miRNAs in PB-MSCs may be used as diagnostic and prognostic indicators in degenerative disorders such $\mathrm{OA}$ and OP. Moreover, changes in relevant expression profiles can be monitored in patients under pharmacological treatment, and provide useful information for therapy follow-up. Circulating miRNAs can be recovered from various body fluids: their altered expression patterns in $\mathrm{OA}$ and $\mathrm{OP}$ are further biomarkers for patients' screening and treatment monitoring. Finally, the potential therapeutic use of PBMSCs in regenerative medicine deserves proper consideration and further investigation.

\section{Funding}

Authors' research was supported by FUR (Fondo Unico della Ricerca) grants of the University of Verona to LDC and MM, respectively.

\section{Author Contribution}

MT Valenti contributed to the conception, design, and data acquisition; drafted and critically revised the manuscript; $M$ Mottes contributed to the conception, design, and data acquisition; drafted and critically revised the manuscript; L Dalle Carbonare contributed to the conception, design, and data acquisition and critically revised the manuscript. All authors gave final approval for publication.

\section{Competing Interests}

The authors declare that they have no competing interests.

\section{References}

1. Macrin D, Joseph JP, Pillai AA, Devi A (2017) Eminent Sources of Adult Mesenchymal Stem Cells and Their Therapeutic Imminence. Stem Cell Rev 13: 741-756. [PubMed]

2. Zvaifler NJ, Marinova-Mutafchieva L, Adams G, Edwards CJ, Moss J, et al (2000) Mesenchymal precursor cells in the blood of normal individuals. Arthritis Res 2: 477-488. [PubMed]

3. Zhou G, Zheng Q, Engin F, Munivez E, Chen Y, et al. (2006) Dominance of SOX9 function over RUNX2 during skeletogenesis. Proc Natl Acad Sci USA 103: 19004-19009. [PubMed]

4. Almalki SG, Agrawal DK (2016) Key transcription factors in the differentiation of mesenchymal stem cells. Differentiation 92: 41-51. [PubMed]

5. Geoffroy V, Kneissel M, Fournier B, Boyde A, Matthias P (2002) High bone resorption in adult aging transgenic mice overexpressing cbfa1/runx2 in cells of the osteoblastic lineage. Mol Cell Biol 22: 6222-6233.

6. Valenti MT, Mottes M, Cheri S, Deiana M, Micheletti V, et al. (2018) Runx2 
Citation: Valenti MT, Mottes M, Carbonare LD (2018) Circulating Progenitor Stem Cells Are Important Biomarkers of Chondrogenesis and Osteogenesis: Employment in Diagnosis and Treatment Follow Up. J Stem Cell Res Ther 8: 425. doi: 10.4172/2157-7633.1000425

overexpression compromises bone quality in acromegalic patients. Endocr Relat Cancer 25: 269-277. [PubMed]

7. Valenti MT, Dalle Carbonare L, Mottes M (2016) Osteogenic Differentiation in Healthy and Pathological Conditions. Int J Mol Sci 18. [PubMed]

8. Simons M, Raposo G (2009) Exosomes--vesicular carriers for intercellular communication. Curr Opin Cell Biol 21: 575-581. [PubMed]

9. Wang X, Omar O, Vazirisani F, Thomsen P, Ekstrom K (2018) Mesenchyma stem cell-derived exosomes have altered microRNA profiles and induce osteogenic differentiation depending on the stage of differentiation. PLoS One: 13. [PubMed]

10. Guilak F, Awad HA, Fermor B, Leddy HA, Gimble JM (2004) Adipose-derived adult stem cells for cartilage tissue engineering. Biorheology 41: 389-399. [PubMed]

11. Barry F, Murphy M (2013) Mesenchymal stem cells in joint disease and repair Nat Rev Rheumatol 9: 584-594.

12. Krawetz RJ, Wu YE, Martin L, Rattner JB, Matyas JR, et al. (2012) Synovial fluid progenitors expressing CD90+ from normal but not osteoarthritic joints undergo chondrogenic differentiation without micro-mass culture. PLoS One 7: e43616.

13. Harris Q, Seto J, O'Brien K, Lee PS, Kondo C, et al. (2013) Monocyte chemotactic protein-1 inhibits chondrogenesis of synovial mesenchymal progenitor cells: an in vitro study. Stem Cells 31: 2253-2265. [PubMed]

14. Fellows CR, Williams R, Davies IR, Gohil K, Baird DM, et al. (2017) Characterisation of a divergent progenitor cell sub-populations in human osteoarthritic cartilage: the role of telomere erosion and replicative senescence. Sci Rep 7: 41421. [PubMed]

15. Campbell TM, Churchman SM, Gomez A, McGonagle D, Conaghan PG, et al. (2016) Mesenchymal Stem Cell Alterations in Bone Marrow Lesions in Patients With Hip Osteoarthritis. Arthritis Rheumatol 68: 1648-1659. [PubMed]

16. Fichadiya A, Bertram KL, Ren G, Yates RM, Krawetz RJ (2016) Characterizing heterogeneity in the response of synovial mesenchymal progenitor cells to synovial macrophages in normal individuals and patients with osteoarthritis. $J$ Inflamm (Lond) 13: 12. [PubMed]

17. Fahy N, de Vries-van ML, Lehmann J, Wei W, Grotenhuis N, et al. (2014) Human osteoarthritic synovium impacts chondrogenic differentiation of mesenchyma stem cells via macrophage polarisation state. Osteoarthritis Cartilage 22: 11671175. [PubMed]

18. Serrano RL, Chen LY, Lotz MK, Liu-Bryan R, Terkeltaub R (2018) Proteasomal function is impaired in human osteoarthritic chondrocytes and this can contribute to decreased SOX9 and aggrecan. Arthritis Rheumatol. [PubMed]

19. Valenti MT, Dalle Carbonare L, Mottes M (2018) Role of microRNAs in progenitor cell commitment and osteogenic differentiation in health and disease (Review). Int J Mol Med 41: 2441-2449. [PubMed]

20. Lian WS, Wu RW, Lee MS, Chen YS, Sun YC, et al. (2017) Subchondral mesenchymal stem cells from osteoarthritic knees display high osteogenic differentiation capacity through microRNA-29a regulation of HDAC4. J Mol Med (Berl) 95: 1327-1340. [PubMed]

21. Karsenty G, Kronenberg HM, Settembre C (2009) Genetic control of bone formation. Annu Rev Cell Dev Biol 25: 629-648. [PubMed]

22. Jing H, Su X, Gao B, Shuai Y, Chen J, et al. (2018) Epigenetic inhibition of Wn pathway suppresses osteogenic differentiation of BMSCs during osteoporosis. Cell Death Dis 9: 176. [PubMed]

23. Liao L, Su X, Yang X, Hu C, Li B, et al. (2016) TNF-alpha Inhibits FoxO1 by Upregulating miR-705 to Aggravate Oxidative Damage in Bone MarrowDerived Mesenchymal Stem Cells during Osteoporosis. Stem Cells 34: 10541067. [PubMed]

24. Koromila T, Baniwal SK, Song YS, Martin A, Xiong J, et al. (2014) Glucocorticoids antagonize RUNX2 during osteoblast differentiation in cultures of ST2 pluripotent mesenchymal cells. J Cell Biochem 115: 27-33.

25. Valenti MT, Dalle CL, Donatelli L, Bertoldo F, Zanatta M, et al. (2008) Gene expression analysis in osteoblastic differentiation from peripheral blood mesenchymal stem cells. Bone 43: 1084-1092. [PubMed]
26. Dalle Carbonare L, Valenti MT, Zanatta M, Donatelli L, Lo Cascio V (2009) Circulating mesenchymal stem cells with abnormal osteogenic differentiation in patients with osteoporosis. Arthritis Rheum 60: 3356-3365.

27. Valenti MT, Garbin U, Pasini A, Zanatta M, Stranieri C, et al. (2011) Role of oxPAPCs in the differentiation of mesenchymal stem cells (MSCs) and Runx2 and PPARgamma2 expression in MSCs-like of osteoporotic patients. PLoS One 6: 3 .

28. Zanatta M, Valenti MT, Donatelli L, Zucal C, Dalle Carbonare L (2012) Runx2 gene expression is associated with age-related changes of bone mineral density in the healthy young-adult population. J Bone Miner Metab 30: 706714. [PubMed]

29. Valenti MT, Mottes M, Biotti A, Perduca M, Pisani A, et al. (2017) Clodronate as a Therapeutic Strategy against Osteoarthritis. Int J Mol Sci 1: 18. [PubMed]

30. Orozco P (2004) Atherogenic lipid profile and elevated lipoprotein (a) are associated with lower bone mineral density in early postmenopausal overweight women. Eur J Epidemiol 19: 1105-1112.

31. Dalle CL, Mottes M, Malerba G, Mori A, Zaninotto M, et al. (2017) Enhanced Osteogenic Differentiation in Zoledronate-Treated Osteoporotic Patients. Int J Mol Sci 1: 18. [PubMed]

32. Nakamura A, Rampersaud YR, Sharma A, Lewis SJ, Wu B, et al. (2016) Identification of microRNA-181a-5p and microRNA-4454 as mediators of face cartilage degeneration. JCI Insight 1: 86820.

33. Xia S, Tian H, Fan L, Zheng J (2017) Peripheral Blood miR-181-5p Serves as a Marker for Screening Patients with Osteoarthritis by Targeting TNFalpha. Clin Lab 63: 1819-1825. [PubMed]

34. Davis C, Dukes A, Drewry M, Helwa I, Johnson MH, et al. (2017) MicroRNA183-5p Increases with Age in Bone-Derived Extracellular Vesicles, Suppresses Bone Marrow Stromal (Stem) Cell Proliferation, and Induces Stem Cell Senescence. Tissue Eng Part A 23: 1231-1240. [PubMed]

35. Kocijan R, Muschitz C, Geiger E, Skalicky S, Baierl A, et al. (2016) Circulating microRNA Signatures in Patients With Idiopathic and Postmenopausa Osteoporosis and Fragility Fractures. J Clin Endocrinol Metab 101: 4125-4134. [PubMed]

36. Hackl M, Heilmeier U, Weilner S, Grillari J (2016) Circulating microRNAs as novel biomarkers for bone diseases - Complex signatures for multifactorial diseases? Mol Cell Endocrinol 432: 83-95.

37. Liew CC, Ma J, Tang HC, Zheng R, Dempsey AA (2006) The peripheral blood transcriptome dynamically reflects system wide biology: a potential diagnostic tool. J Lab Clin Med 147: 126-132. [PubMed]

38. Valenti MT, Mori A, Malerba G, Dalle Carbonare L (2015) Mesenchymal stem cells: A new diagnostic tool? World journal of stem cells 7: 789. [PubMed]

39. Granero-Molto F, Weis JA, Miga MI, Landis B, Myers TJ, et al. (2009) Regenerative effects of transplanted mesenchymal stem cells in fracture healing. Stem Cells 27: 1887-1898.

40. Ranzoni AM, Corcelli M, Hau KL, Kerns JG, Vanleene M, et al. (2016) Counteracting bone fragility with human amniotic mesenchymal stem cells. Sci Rep 6: 1.

41. Gotherstrom C, Westgren M, Shaw SW, Astrom E, Biswas A, et al. (2014) Preand postnatal transplantation of fetal mesenchymal stem cells in osteogenesis imperfecta: a two-center experience. Stem Cells Transl Med 3: 255-264. [PubMed]

42. Prockop DJ (2017) The exciting prospects of new therapies with mesenchymal stromal cells. Cytotherapy 19: 1-8. [PubMed]

43. Wan C, He Q, Li G (2006) Allogenic peripheral blood derived mesenchymal stem cells (MSCs) enhance bone regeneration in rabbit ulna critical-sized bone defect model. J Orthop Res 24: 610-618.

44. Alm JJ, Koivu HM, Heino TJ, Hentunen TA, Laitinen S, et al. (2010) Circulating plastic adherent mesenchymal stem cells in aged hip fracture patients. J Orthop Res 28: 1634-1642. [PubMed]

45. Ramirez M, Lucia A, Gomez-Gallego F, Esteve-Lanao J, Perez-Martinez A, et al. (2006) Mobilisation of mesenchymal cells into blood in response to skeletal muscle injury. Br J Sports Med 40: 719-722. [PubMed] 